\title{
The curious case of peroxiredoxin-5: what its absence in aves can tell us and how it can be used
}

\author{
Marc Pirson(1), André Clippe and Bernard Knoops
}

\begin{abstract}
Background: Peroxiredoxins are ubiquitous thiol-dependent peroxidases that represent a major antioxidant defense in both prokaryotic cells and eukaryotic organisms. Among the six vertebrate peroxiredoxin isoforms, peroxiredoxin-5 (PRDX5) appears to be a particular peroxiredoxin, displaying a different catalytic mechanism, as well as a wider substrate specificity and subcellular distribution. In addition, several evolutionary peculiarities, such as loss of subcellular targeting in certain species, have been reported for this enzyme.

Results: Western blotting analyses of 2-cys PRDXs (PRDX1-5) failed to identify the PRDX5 isoform in chicken tissue homogenates. Thereafter, via in silico analysis of PRDX5 orthologs, we went on to show that the PRDX5 gene is conserved in all branches of the amniotes clade, with the exception of aves. Further investigation of bird genomic sequences and expressed tag sequences confirmed the disappearance of the gene, though TRMT112, a gene located closely to the $5^{\prime}$ extremity of the PRDX5 gene, is conserved. Finally, using in ovo electroporation to overexpress the long and short forms of human PRDX5, we showed that, though the gene is lost in birds, subcellular targeting of human PRDX5 is conserved in the chick.

Conclusions: Further adding to the distinctiveness of this enzyme, this study reports converging evidence supporting loss of PRDX5 in aves. In-depth analysis revealed that this absence is proper to birds as PRDX5 appears to be conserved in non-avian amniotes. Finally, taking advantage of the in ovo electroporation technique, we validate the subcellular targeting of human PRDX5 in the chick embryo and bring forward this gain-of-function model as a potent way to study PRDX5 functions in vivo.
\end{abstract}

Keywords: Peroxiredoxin-5, Aves, Birds, Amniotes, Vertebrates, TRMT112, Gene loss, Gain-of-function

\section{Background}

Reactive oxygen and nitrogen species (ROS/RNS) are toxic compounds generated out of the cell, but also intracellularly through the electron transport chain, and in various physiological and pathophysiological situations. Their toxicity arises from unpaired electrons, or the oxygen and nitrogen content within these molecules that render them highly reactive towards biological components such as DNA, lipids and proteins [1]. However, at moderate concentrations ROS/RNS have also been shown to be implicated in numerous cellular processes, including cell signaling $[2,3]$. Due to this dual role, fine

\footnotetext{
*Correspondence: bernard.knoops@uclouvain.be

Group of Animal Molecular and Cellular Biology, Institut des Sciences de la

Vie (ISV), Université catholique de Louvain, 4-5 Place Croix du Sud, 1348

Louvain-la-Neuve, Belgium
}

control of ROS/RNS concentrations at the cellular level is essential, a function performed by the different enzymatic and non-enzymatic antioxidant systems cells are equipped with. In particular, thiol-dependent peroxidases peroxiredoxins (PRDXs) have been reported to be major antioxidant enzymes in prokaryotes and eukaryotic cells due to their abundance and high catalytic efficiency for reducing peroxides [4].

In most vertebrates, the PRDX family comprises 6 enzymes (PRDX1-6) divided into three subgroups depending on their catalytic mechanism and the conservation of their catalytic cysteines: typical 2-Cys PRDXs (PRDX1-4), atypical 2-Cys PRDX (PRDX5) and 1-Cys PRDX (PRDX6) [5, 6]. While all PRDX isoforms reduce peroxides via the nucleophilic attack of their peroxidatic cysteine $(\mathrm{Cp})$, leading to the formation of a sulfenic acid 
(Cp-SOH), the resolution step of the catalytic cycle differs for each subgroup. The oxidized $\mathrm{Cp}$ forms a disulfide bond with the resolution cysteine $(\mathrm{Cr})$ of another PRDX subunit for typical 2-Cys PRDXs, or with the $\mathrm{Cr}$ of the same enzymatic subunit for atypical 2-cys PRDX5 [7]. For the 1-cys PRDX6, reduction of the Cp sulfenic acid occurs through condensation with an external thiol, generally glutathione in the presence of glutathione-Stransferase (GST) $\pi$ [8-10].

With regards to other PRDXs, PRDX5 appears to be a particular PRDX whose cytoprotective function is well documented. Indeed, in addition to its different structure and catalytic mechanism, human PRDX5 shows wider substrate specificity, reducing hydrogen peroxide, but also alkyl hydroperoxides and peroxynitrite, and a broad subcellular distribution, localizing to the mitochondria, the cytosol, the peroxisome and, in some situations, to the nucleus [11]. These multiple localizations are a consequence of the existence of two PRDX5 isoforms encoded by a single PRDX5 gene containing alternative transcription start sites and two in-frame translation initiation sites [12]. The short form of PRDX5 (S-PRDX5) will be found in the cytosol and nucleus, but also in peroxisomes thanks to a weak carboxyterminal peroxisomal targeting sequence type 1 (PTS1). The long form of PRDX5 (L-PRDX5) contains an amino-terminal mitochondrial targeting sequence (MTS) which is cleaved after mitochondrial import, producing a mature mitochondrial PRDX5 identical to the short form [12-14]. PRDX5 is an evolutionarily conserved enzyme, PRDX5 orthologs existing throughout the animal kingdom, in invertebrates and vertebrates alike $[12,15,16]$. However, although the short form is conserved, recent reports have shown that mitochondrial targeting of PRDX5 is absent in certain mammalian species, including pig and canids $[15,16]$.

Here we report converging evidence pointing to the loss of the PRDX5 gene in birds though it is conserved in nonavian amniotes, and lead a thorough discussion concerning the potential biological significance of this absence. Finally, using in ovo electroporation, we show that subcellular targeting of human PRDX5 is functional in chick spinal cord and we validate the chick as a novel gain-of-function model for studying PRDX5 function in vivo.

\section{Methods \\ Computer analysis}

Human PRDX5 and TRMT112 mRNA (PRDX5: NM_01 2094.4; TRMT112: NM_001286082.1), and protein (PRD X5: NP_036226.1; TRMT112: NP_001273011.1) sequences were obtained from National Center for Biotechnology Information (NCBI). Identification of predicted PRDX5 and TRMT112 orthologs in other species was carried out using NCBI Blast, as well as UCSC and International Crocodilian Genomes Working Group (ICGWG) genome browsers [17]. Translation of nucleotide sequences was performed via the ExPaSy translate tool [18]. Multiple sequence alignment was done with Clustal Omega [19], and displayed using GeneDoc with the conserved residue shading mode and similarity groups enabled [20]. TargetP 1.1. [21, 22] and Mitoprot [23] were used for mitochondrial targeting prediction.

\section{Antibodies}

Primary antibodies used in the present study were previously validated and described [24, 25]. References of the antibodies, as well as dilutions used for Western blotting and immunofluorescence analyses are specified in Table 1 . Mouse monoclonal antibodies anti-ATP synthase subunit beta (ATPB; 3D5, Abcam) and anti-catalase (CAT; CAT505, Sigma) were used as markers of mitochondria and peroxisomes, respectively. Secondary antibodies Alexa Fluor 546 Donkey anti-Mouse IgG and Alexa Fluor 633 Donkey anti-Goat IgG were obtained from Invitrogen. The background signal generated by the secondary antibodies was tested and considered negligible.

\section{Animal and tissue processing}

Chicken tissue was obtained from an adult male red junglefowl (Gallus gallus), kindly provided by a farm in Tilly (Belgium). Owner of the animal verbally consented to its use in the present study. Fertilized eggs were obtained from Wyverkens farm (Halle, Belgium). Animal and tissue processing was performed according to protocols described in Pirson and Knoops (2015) and Pirson et al. (2015) [25, 26].

Table 1 Primary antibodies

\begin{tabular}{lll}
\hline Antigen & Source & Dilution \\
\hline PRDX1 & Rabbit polyclonal, obtained in Hormonology laboratory of Marloie (Belgium), No. UC232 & $1: 4000$ for Western blotting \\
PRDX2 & Rabbit polyclonal, obtained in Hormonology laboratory of Marloie (Belgium), No. UC197 & $1: 5000$ for Western blotting \\
PRDX3 & Rabbit polyclonal, obtained in Hormonology laboratory of Marloie (Belgium), No. UC210 & $1: 5000$ for Western blotting \\
PRDX4 & Rabbit polyclonal, obtained in Hormonology laboratory of Marloie (Belgium), No. UC194 & $1: 5000$ for Western blotting \\
PRDX5 & Rabbit polyclonal, obtained in Hormonology laboratory of Marloie (Belgium), No. G234 & $1: 1500$ for immunofluorescence \\
& & $1: 5000$ for Western blotting \\
ATPB & Abcam; catalog No. ab14730, mouse monoclonal (3D5) & $1: 500$ for immunofluorescence \\
CAT & Sigma; catalog No. C0979, mouse monoclonal (CAT-505) & $1: 100$ for immunofluorescence \\
\hline
\end{tabular}




\section{Western blotting}

Western blotting of chicken tissue homogenate proteins and SH-SY5Y (human neuroblastoma) cell lysate proteins, serving as a positive control, was performed as previously described $[25,26]$.

\section{Cloning of human L-PRDX5 and S-PRDX5 CDNA}

Cloning and purification of human L-PRDX5 and S$P R D X 5$ cDNA were performed as described previously [27]. The human PRDX5 cDNA was PCR-amplified with forward primer 5'-GGCCGTGAATTCGGTATGGGAC TAGCTGGC-3' (EcoRI site is in italics) for $L-P R D X 5$ or 5' -AGAGCCGAATTCGCCATGGCCCCAATCAAG-3'

(EcoRI site is in italics) for S-PRDX5 and reverse primer $5^{\prime}$ TAATCTGCGGCCGCGCCTCAGAGCTGTGAGAT-3'

(NotI site is in italics). After restriction, the sequence was ligated into pCMS-eGFP vector (Clontech, Palo Alto, CA, USA) in which $L-P R D X 5$ or S-PRDX5 coding sequences are under the control of a CMV promoter.

\section{In ovo electroporation}

Treatment of fertilized eggs and in ovo electroporation of chick embryos were performed as previously described [26]. Briefly, pCMS-eGFP vector (Empty vector), pCMS-eGFP vector with human L-PRDX5 (L-PRDX5 vector) or $S$-PRDX5 (S-PRDX5 vector) cDNA sequences were injected into the neural tube of Hamilton-
Hamburger stage $(\mathrm{HH}) 12-14$, i.e. embryonic day $2-2.5$ (E2-2.5), chick embryos at a concentration of $2 \mu \mathrm{g} / \mu \mathrm{l}$ prior to electroporation. Subsequent processing (cryosectioning and immunolabeling) was carried out $48 \mathrm{~h}$ after electroporation, on stage E4-4.5 embryos.

\section{Immunofluorescence assay}

Immunofluorescence assay and image acquisition of chick embryonic spinal cords was performed as previously described $[25,26]$.

\section{Results}

\section{Western blotting analysis of PRDXs in chicken tissue homogenates}

The PRDX5 gene is conserved throughout evolution and orthologs have been described in a wide array of animal species, ranging from invertebrates to mammals $[12,15$, 16]. In chicken (Gallus gallus), however, though the existence of PRDX1, PRDX3-4, and PRDX6 isoforms was previously reported, the possible absence of typical 2Cys PRDX2 and atypical 2-Cys PRDX5 was not discussed [28]. Here we show that absence of PRDX5 in Gallus gallus is further supported by Western blotting analyses of chicken lung, heart, liver, and muscle homogenates using antibodies directed towards human 2-Cys PRDX1-5 (Fig. 1). Soluble proteins of human SH-SY5Y cells were used as a positive control.

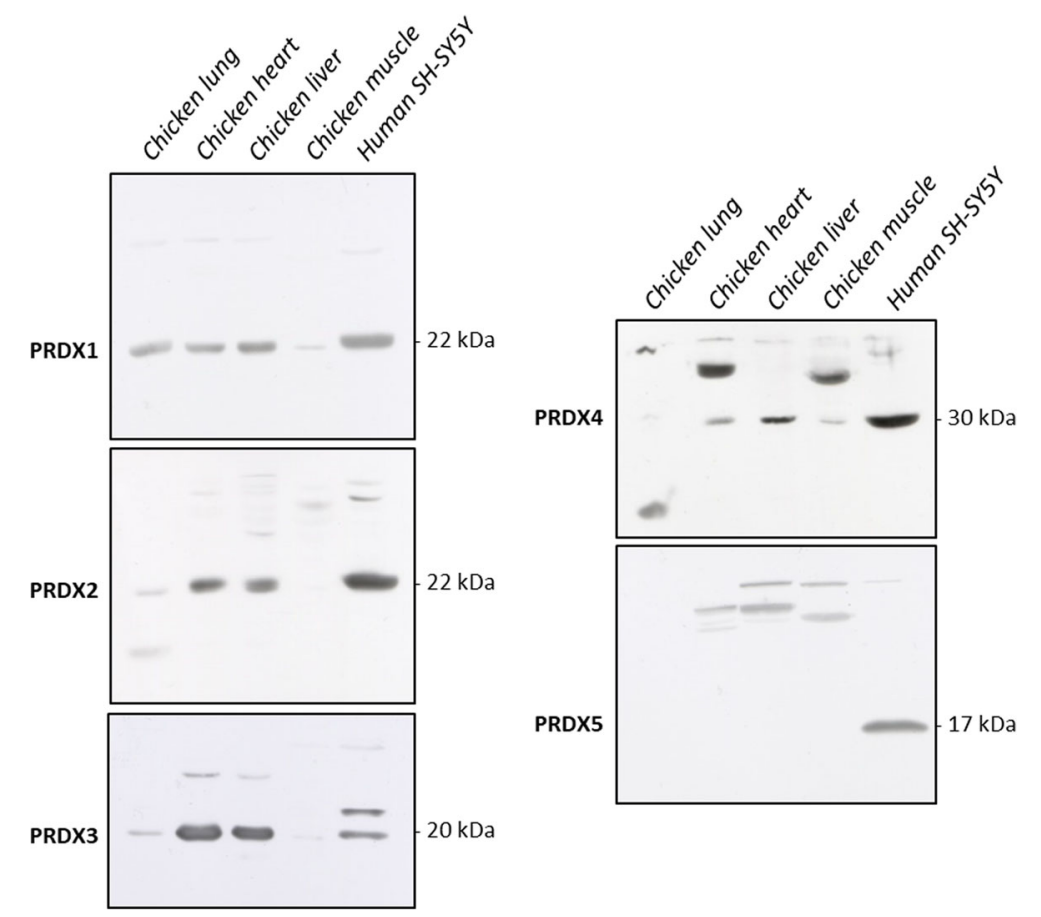

Fig. 1 Western blotting analysis of 2-Cys PRDXs in chicken tissue. Proteins from chicken lung, heart, liver, and muscle homogenates and human neuroblastoma SH-SY5Y cell lysates (positive control) were loaded on a SDS-PAGE gel and probed with antibodies directed to 2-Cys PRDXs (see Table 1). Molecular weights $(\mathrm{kDa})$ are indicated 
All antibodies, including anti-PRDX2, yielded a band at the expected molecular weight, with the exception of anti-PRDX5 which produced no signal around the $17 \mathrm{kDa}$ mark. Higher molecular weight bands observed for PRDX3 and PRDX4 were around the $40 \mathrm{kDa}$ and $60 \mathrm{kDa}$ marks and likely correspond to the dimerized form of the enzyme resulting from incomplete reduction of the samples prior to the analysis. The low intensity high molecular weight bands detected for several of the enzymes, including PRDX5, were deemed to be linked to aspecific binding of the antibody.

\section{Identification of PRDX5 orthologs in amniotes}

In order to further evidence the loss of PRDX5 in birds during evolution and determine whether the enzyme is conserved in non-avian amniotes, an in silico examination of PRDX5 orthologs was carried out and amino acid sequence alignment was performed (Fig. 2). On June 22nd 2017, reference mRNA and protein sequences for Homo sapiens (human) PRDX5 were obtained from NCBI sequence databases. These sequences were used to identify predicted orthologs in bats (Pteropus alecto, black flying fox), lizards (Anolis carolinensis, anole lizard), turtles (Chrysemys picta bellii, painted turtle), snakes (Python bivittatus, Burmese python), crocodiles (Alligator mississippiensis, American alligator), and amphibians (Xenopus laevis, african clawed toad) using the blastx function (search a protein database using a translated nucleotide query) on non-redundant protein sequence database. Conversely, this analysis did not yield sequences of considerable homology in aves. Similarly, tblastn analysis (search a translated nucleotide database using a protein query) of

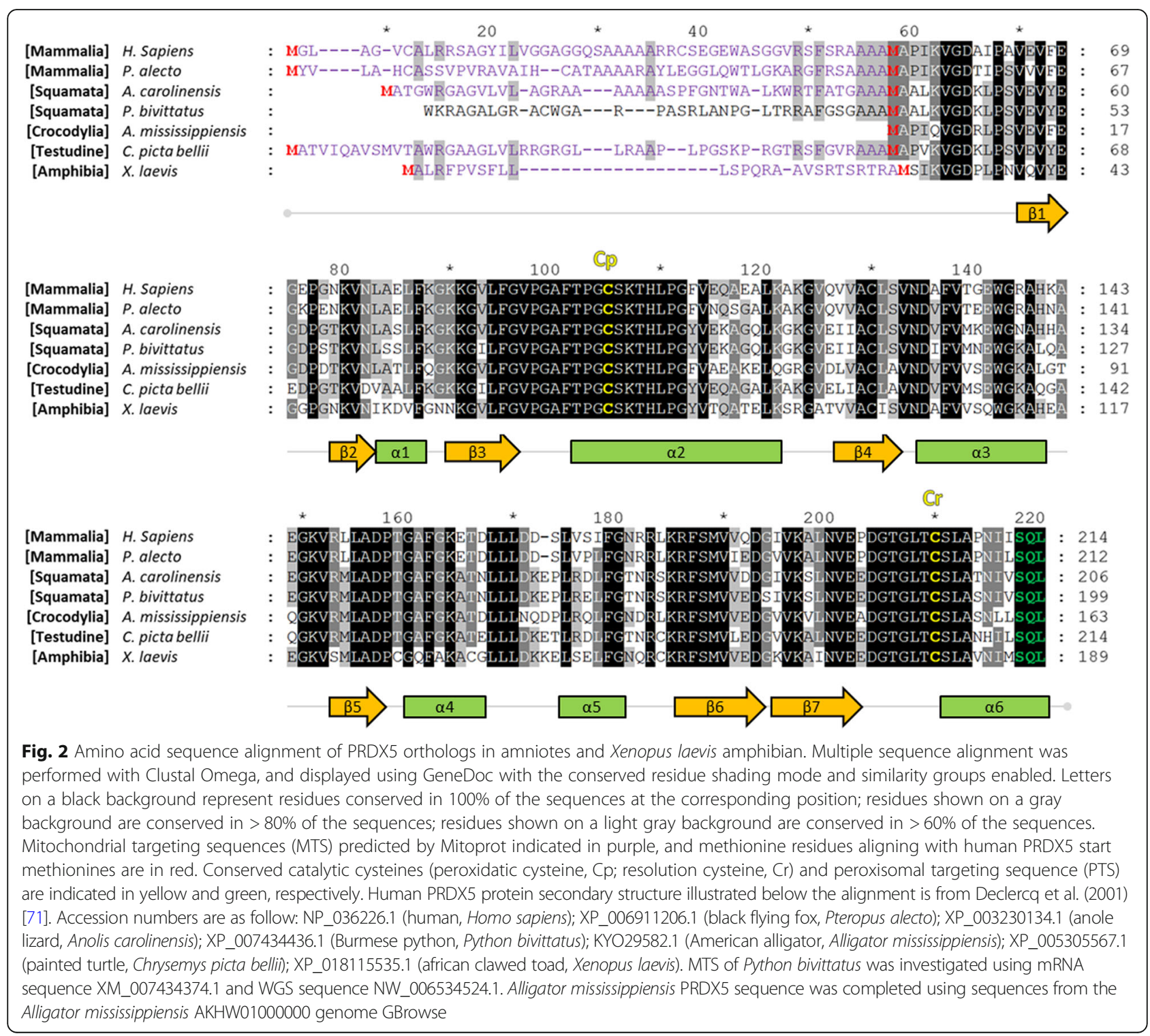


predicted Anolis carolinensis (anole lizard) PRDX5 protein sequence on all available NCBI databases containing bird sequences generated no potential candidates for PRDX5.

All identified orthologs displayed conserved peroxidatic cysteine $(\mathrm{Cp})$, resolution cysteine $(\mathrm{Cr})$ residues and surrounding amino acids, as well as the peroxisomal targeting sequence type 1 (PTS1) at the carboxy-terminus (Fig. 2). Mitochondrial targeting sequences (MTS) were identified in Pteropus alecto, Anolis carolinensis, Chrysemys picta bellii and Xenopus laevis, but could not be highlighted in Python bivittatus or Alligator mississippiensis predicted PRDX5 amino acid sequences. To investigate this, analysis of the genomic sequences from which these predicted PRDX5 protein sequences originate was performed. For Python bivittatus, a sequence upstream of the start methionine of S-PRDX5 showed strong homology to Anolis carolinensis PRDX5 MTS but was not considered as an open reading frame because of indeterminations at the $5^{\prime}$ extremity of the nucleotide sequence. Concerning genomic sequence of Alligator mississippiensis containing the PRDX5 coding sequence, a sequence $5^{\prime}$ to the $S-P R D X 5$ start codon reveals the presence of an in-phase STOP codon. Similarly, in-phase STOP codons were also present upstream of the $S$ PRDX5 in two other crocodilian species, Crocodylus porosus and Gavialis gangeticus (data not shown).

\section{TRMT112 gene is conserved in birds}

The absence of PRDX5 in available Gallus gallus sequences could be linked to rearrangements affecting syntenic gene cluster or due to gaps or errors in genomic sequences available for birds. In order to examine these possibilities, avian orthologs of TRMT112, a gene located closely to the $5^{\prime}$ extremity of $L-P R D X 5$ gene in mammals [15], were identified (Fig. 3). To this end, on June 22nd 2017, human TRMT112 mRNA and protein sequences were used to identify orthologs in birds by blastp (search a protein database using a protein query) on non-redundant protein databases for aves and tblastn on expressed tag sequence (EST) databases for aves. Predicted TRMT112 orthologous sequences were identified for several bird species including Gallus gallus (red junglefowl), Sturnus vulgaris (common starling), and Parus major (great tit). Inspection of genomic regions adjacent to TRMT112 gene revealed no trace of the PRDX5 gene in these species (data not shown).

\section{Overexpression of human L-PRDX5 and S-PRDX5 in Gallus gallus}

Though cellular models such as cancerous cell lines are a useful tool to study protein function, these models are generally oversimplifications of actual biological systems as they do not account for the complex cellular composition, interactions, and environment that exist in vivo and may fail to reproduce key physiological processes. For this reason, the use of in vivo models is critical to confirm data obtained in cellular or in vitro models, and obtain a clearer view of protein function in the context of a live tissue or organism. Although in vivo models, including knockout and transgenic mice models, exist for all typical 2-cys PRDXs (Prdxs1-4) and 1-cys PRDX6, and have offered precious clues to understanding the function of these enzymes, so far no such models have been published for PRDX5 [29-31]. Thanks to technical advances in chick embryo genetic manipulation, the absence of PRDX5 gene in birds opens the door to the development of an interesting gain-of-function model to study both long and short forms of PRDX5. To this end,

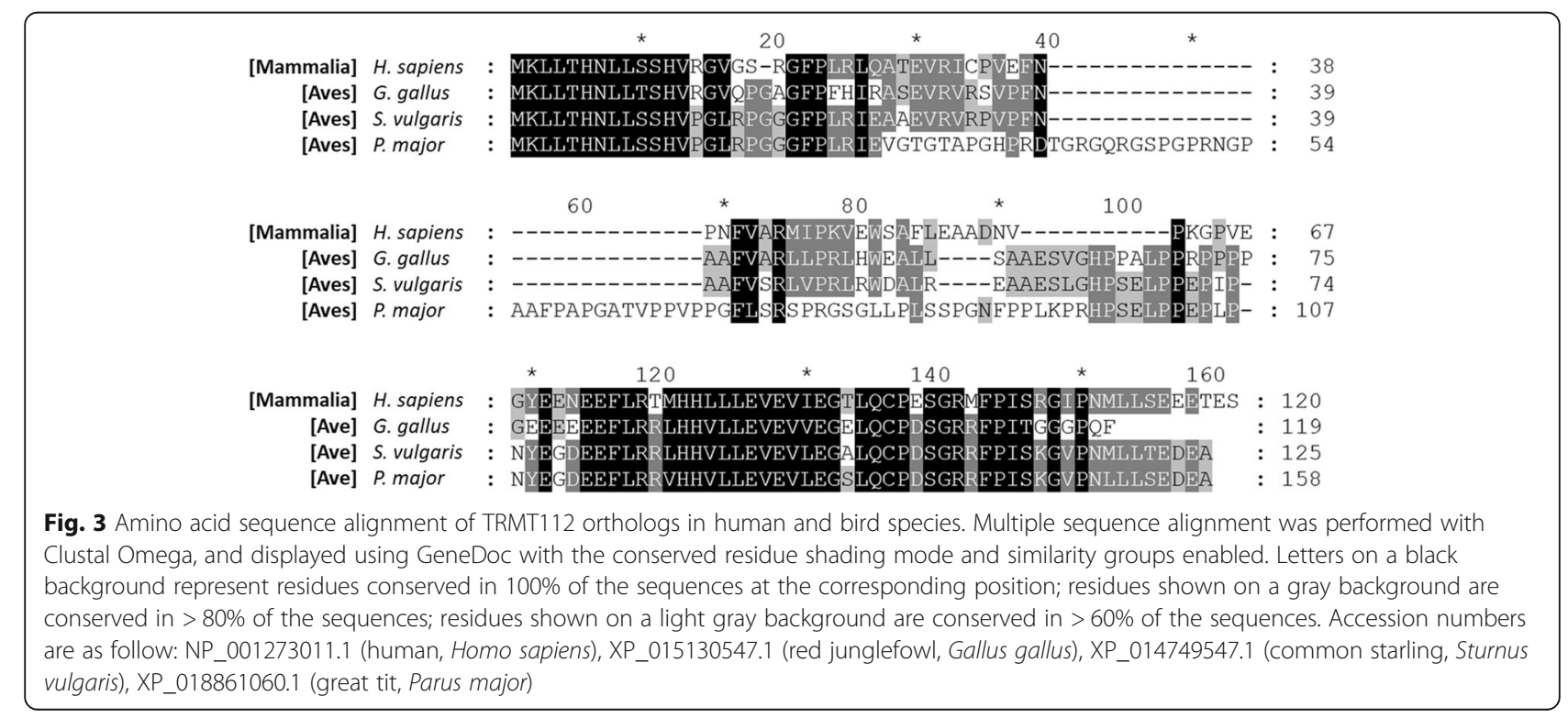


injection of DNA constructs containing cDNA sequences coding for human L-PRDX5 and S-PRDX5 into the neural tube and in ovo electroporation were performed on stage HH12-14 chick embryos (E2-E2.5). Forty-eight hours after electroporation, immunofluorescence assay using polyclonal antibody directed to human PRDX5 revealed intense signal in the ipsilateral (electroporated) as opposed to the contralateral (non-electroporated) side of the spinal cord in embryos electroporated with both $L-P R D X 5$ vector and $S$-PRDX5 vector (Fig. 4ab). Concordantly with these results, electroporation of the empty vector did not produce any immunostaining associated to PRDX5 (Fig. 4b). In order to determine whether subcellular targeting of human L-PRDX5 and S-PRDX5 is conserved in Gallus gallus and further validate our gain-of-function model, colocalization analysis of overexpressed PRDX5 and subcellular markers were performed on electroporated chick embryos $48 \mathrm{~h}$ after electroporation (E4.5) (Fig. 5). Electroporation of $L$ PRDX5 vector yielded a punctuated cytoplasmic staining for PRDX5 that colocalized with mitochondrial marker ATP synthase subunit beta (ATPB) (Fig. 5a-e). No overlap of L-Prdx 5 and peroxisomal catalase (CAT) signals was observed (Fig. 5f-j). Chick embryos electroporated with S-PRDX5 vector showed homogenous PRDX5 immunoreactivity in cytoplasm, but also in nucleus. Staining colocalized with peroxisomal marker CAT, however, accumulation in the organelle was not obvious (Fig. 5p-t). Though the signals associated to S-PRDX5 and mitochondrial marker ATPB might overlap to a certain extent, this was not systematic in electroporated cells and might be artefactual (Fig. 5k-o).

\section{Discussion}

PRDX5 is lost in aves but conserved in non-avian amniotes

The major observation made in this work is the loss of the PRDX5 gene in birds during evolution, despite the fact that orthologous PRDX5 enzymes are described or predicted in non-avian amniotes, including reptiles, such as the anole lizard, the painted turtle, the Burmese python, and the American alligator, as well as human and flying mammal Pteropus Alecto.

This conclusion is supported by several converging lines of evidence. First, Western blotting of chick tissue homogenates with antibodies directed against 2-Cys
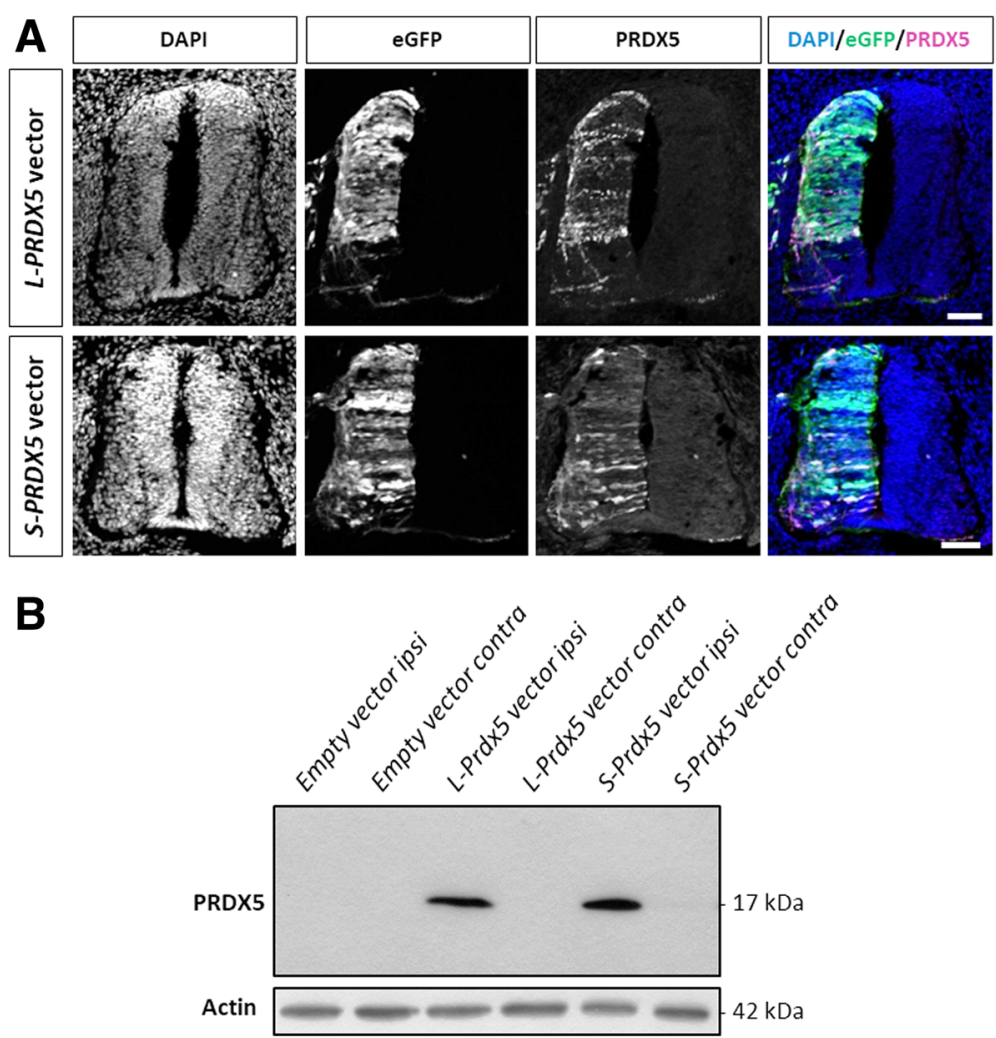

Fig. 4 Expression of L-PRDX5 and S-PRDX5 by in ovo electroporation. a. Immunofluorescence detection of PRDX5 in E4.5 chick embryo spinal cords electroporated with L-PRDX5 and S-PRDX5 vectors. eGFP signal serves as control of effective plasmid electroporation. Nuclear DAPI staining is used to visualize cells in the tissues. Scale bars $=50 \mu \mathrm{m}$. b. Western blotting analysis of the contralateral (non-electroporated) and ipsilateral (electroporated) sides of dissected spinal cords of E4.5 chick embryos electroporated with empty vector, L-PRDX5 vector, or S-PRDX5 vector 


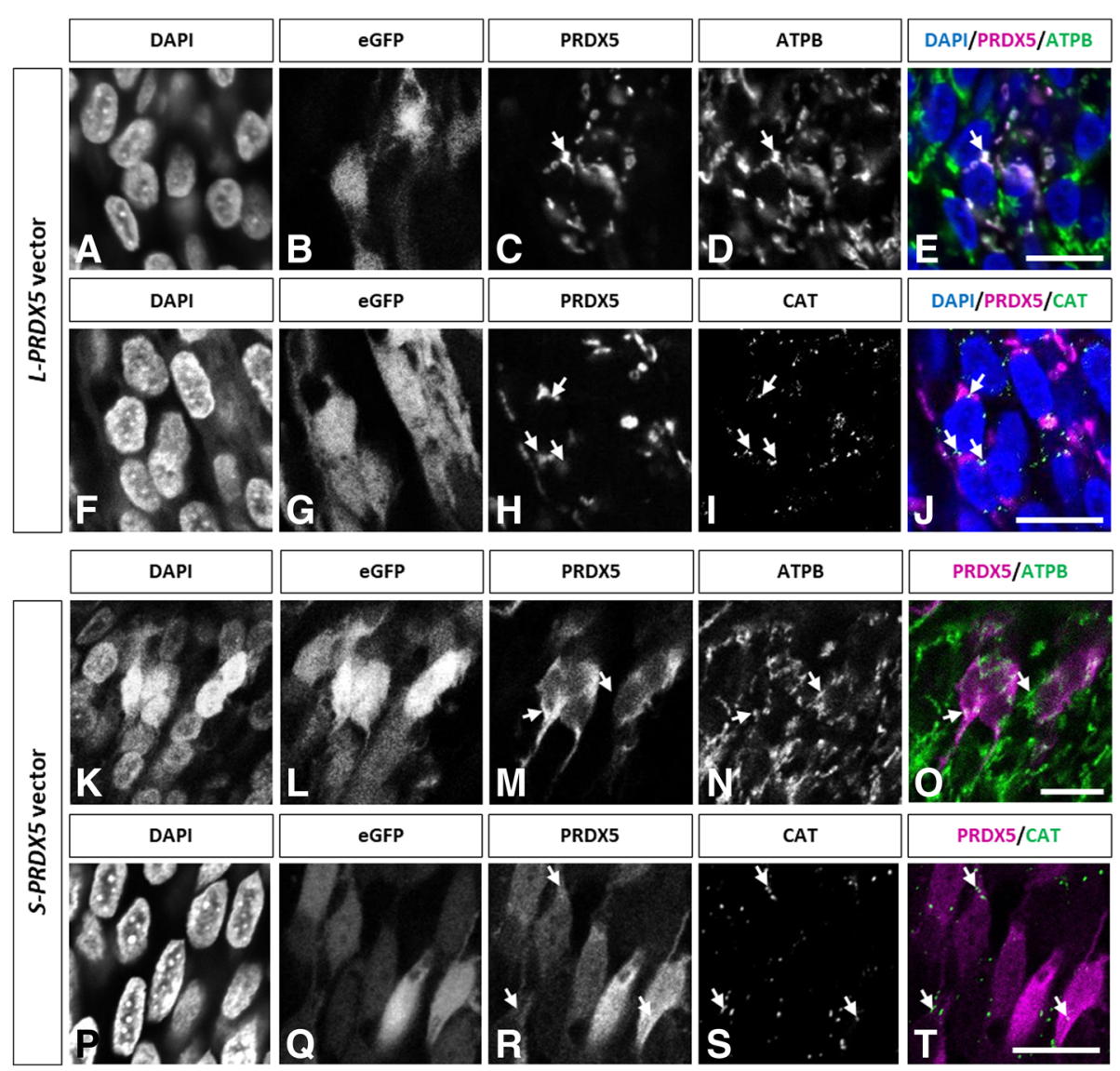

Fig. 5 Subcellular localization of L-PRDX5 and S-PRDX5 in chick spinal cord electroporated with L-PRDX5 and S-PRDX5 vectors. Co-immunodetection of L-PRDX5 (a-j) and S-PRDX5 (k-t) with mitochondrial marker ATPB and peroxisomal marker CAT in cryosections of embryonic chick spinal cord. eGFP signal serves as control of effective plasmid electroporation. DAPI staining is used to visualize cell nucleus. Scale bars $=10 \mu \mathrm{m}$

PRDXs highlighted the presence of bands at expected molecular weights for all PRDXs except PRDX5. This comes as a surprise for the antibody directed to PRDX2 as this enzyme is also considered absent in chicken [28]. However, high degree of homology between human PRDX1-2 used for generation of the antibodies might have led to aspecific binding of the anti-PRDX2 antibody with chicken PRDX1. It is of note that failure of the anti-PRDX5 antibody to recognize an orthologous PRDX5 in chick tissue seems unlikely considering that it is a polyclonal antibody and that PRDX5 orthologs share important sequence homology ( $>69 \%$ identity in the amino acid sequence between the human protein used to generate the antibody and the PRDX5 of species considered in Fig. 2). Indeed, anti-PRDX5 antibody used in this work has been shown to detect PRDX5 in several other species including mouse, rat, pig, dog, and African clawed toad [15, 16, 24, 32].

To further support the hypothesis that the PRDX5 gene is absent in chicken, and birds in general, and determine whether the enzyme is present in other amniotes, an in silico search was carried out to identify
PRDX5 orthologs in different amniote species. Blast screening of available bird sequences in NCBI databases did not enable us to highlight the existence of an avian PRDX5 gene. Conversely, PRDX5 orthologs were identified in several sauropsida species, including anole lizard, painted turtle, and American alligator, suggesting disappearance of PRDX5 occurred after the bird-lizard split about 254 million years ago (Triassic period) [33]. Determination of a more precise timeframe would require sequence data of now extinct species.

A major criticism of the identification of genes in this way is the dependence of the methodology on complete and reliable genomic sequences and expressed tag sequence (EST) libraries. Moreover, bird genomes are known to be smaller compared to that of other amniotes [34]. This reduction in genome size has been associated to loss of non-coding DNA but also of protein coding genes. Indeed, chicken genome comprises about 15,500 genes compared to approximately 20,800 in human and 18,600 in the anole lizard [35]. Furthermore, the important loss of protein coding genes during evolution of the avian genome seems linked to high rates of chromosomal 
rearrangements affecting syntenic blocks of genes [3436]. For these reasons, identification of TRMT112 (previously named HSPC152), a gene closely located to the PRDX5 gene, was carried out. No TRMT112 ortholog could initially be identified from Gallus gallus genomic sequences, suggesting the TRMT112-PRDX5 locus could be subject to gaps, errors, or indeterminations. However, genomic sequences corresponding to TRMT112 were identified in the common starling and the great tit, and mRNAs corresponding to TRMT112 were highlighted in chicken suggesting that genes located in this locus can effectively be identified in aves. Moreover, analysis of the sequences situated in the vicinity of the TRMT112 gene in these species further supports that birds are indeed devoid of the PRDX5 gene.

The absence of PRDX5 in birds suggests that this enzyme is not essential to these species and the evolutionary success encountered by the avian class does not seem to have been affected by this loss. This highlights questions concerning redox regulation and oxidative stress response in aves. Interestingly, the striking longevity displayed by birds compared to mammals of equivalent mass has, in part, been attributed to increased resistance to oxidative stress or reduced oxidative damages [37, 38]. Indeed, birds have been shown to be more resistant to lipid peroxidation than rodents due to the different composition of their biological membranes [39-41]. Ogburn et al. (1998) also demonstrated that, in oxidative stress conditions, bird epithelial kidney cells exhibited less DNA damage compared to that of rodents [41]. Furthermore, bird mitochondria appear to produce less hydrogen peroxide than rodent mitochondria [42-44]. Due to the physiological adaptations associated to the evolution of flight, bats display similarities to birds with regards to oxidative stress, a fact which has been linked to their remarkable longevity [38]. Indeed, contrasting with their high metabolic rates, both bats and birds exhibit reduced reactive species production in basal and stimulated conditions, as well as increased resistance to oxidative damage compared to non-flying mammals [38, 45, 46]. Along these lines, flight driven aerobicity has been proposed to act as an oxidative sink wherein mitochondria, due to their energetic metabolism and elevated antioxidant defences, would actually be consumers rather than producers of reactive species [37]. Due to these adaptations, it could be hypothesized that PRDX5 may no longer have been essential to antioxidant defence in these species. Conversely to birds, PRDX5 was readily identified in black flying fox suggesting that the physiological characteristics associated to both birds and bats are not sufficient to explain the disappearance of PRDX5. However, evolutionary divergence of birds largely predates that of bats and physiological changes leading to reduced oxidative damages might be less important in bats than in birds [47]. Moreover these species might have followed different evolutionary paths in order to cope with the adaptations.

Another explanation to the loss of PRDX5 could reside in functional redundancy with other antioxidant enzymes. Indeed several other peroxide reductases, including several PRDXs, catalase, or members of glutathione peroxidase family, are conserved in birds [28, 48-51]. Interestingly, lower activity of several antioxidant enzymes and molecules has been reported in bird compared to rodent brain, lung, and liver suggesting that birds might not need the same degree of antioxidant defence [52-54]. Therefore, the disappearance of PRDX5 in birds might be the consequence of functional redundancy with regards to other antioxidant enzymes, as well as reduced necessity for birds to protect themselves from oxidative damage.

It is worth mentioning that, in addition to its wellstudied cytoprotective function, other roles have been attributed to PRDX5, notably in immune and inflammatory processes. Indeed, PRDX 5 has been reported to be overexpressed in immunostimulated macrophages [55, 56], while extracellular PRDX5 fragments were shown to induce macrophage infiltration via toll-like receptor activation [57]. Moreover, PRDX5 has been negatively linked to pro-inflammatory JNK signaling leading to decreased resistance to infection in drosophila [58]. Coincidentally, the immune system of birds appears to be quite different to that of mammals. Kaiser et al. (2005) reported the production of different cytokines and chemokines during immune response in chicken compared to other vertebrates suggesting distinct immune mechanisms in these species [59]. Moreover, birds display reduced immune gene repertoires compared to mammals [60]. Therefore, the function of PRDX5 in mammalian immune response may no longer have been required in the context of avian immunity and thus the gene ceased undergoing selection.

\section{Crocodilian PRDX5 lacks a functional mitochondrial targeting sequence}

An anecdotal finding of this work is the abolition of the MTS of Alligator PRDX5. Indeed, analysis of the sequence located upstream of the start methionine of SPRDX5 highlighted the presence of a translation STOP in the MTS. PRDX5 mitochondrial targeting is conserved in human, black flying fox, anole lizard, Burmese python, African clawed toad, as well as numerous other vertebrate and invertebrate species [13, 16, 61-67]. However, disruption of mitochondrial targeting of PRDX5 is thought to have occurred on at least two separate occasions during mammalian evolution, namely in pig and canids. In addition, expression of the mitochondrial isoform of PRDX5 in canine MDCK cells had 
deleterious consequences to the cell upon peroxide exposure $[15,16]$. It is possible that L-PRDX5 began exhibiting a similar cytotoxic effect in the crocodile lineage and that mitochondrial targeting was therefore counter-selected.

Although functional MTS could not be identified in Burmese python due to indeterminations in the genomic sequence, the partial sequence obtained showed strong homology with the MTS of the anole lizard. This suggests that mitochondrial targeting of PRDX5 is indeed conserved in snakes as loss of MTS functionality would likely have led to numerous mutations in this sequence should it no longer be submitted to selective pressure. It is important to note that this result is based solely on currently available sequence data and remains speculative until the mitochondrial targeting of PRDX5 in snakes and crocodiles is verified experimentally. Also, it is worth mentioning that the PTS1 is conserved in all PRDX5 sequences analyzed in this study.

\section{A new in vivo PRDX5 gain-of-function model}

In addition to being interesting from an evolutionary standpoint, the absence of PRDX5 in birds also offers the opportunity to develop several gain-of-function models to study PRDX5. Though a cell culture approach through commonly used DT40 chicken B cell line could be envisioned, over the last decade, in ovo electroporation has been widely used for gain- or loss-of-function studies during chick embryonic development $[26,68]$. This is especially interesting in light of recent reports of a particular expression pattern of PRDX5 in the mouse embryonic spinal cord [25].

In the present study, with the aim of developing the in ovo electroporation of PRDX5 model, DNA constructs coding for long and short isoforms of human PRDX5 were electroporated into the embryonic chick spinal cord. Both L-PRDX5 and S-PRDX5 were successfully overexpressed as shown by the strong immunostaining observed on the ipsilateral (electroporated) side of the spinal cord as opposed to the contralateral (non-electroporated) side. Moreover, localization of these forms of the enzyme was coherent with expected subcellular targeting. Indeed, $L-P R D X 5$ vector produced signal that specifically colocalized with mitochondrial marker ATPB while the S-PRDX5 vector generated staining in the cytosol, nucleus, and peroxisomes as highlighted through colocalization with catalase. Though our results suggest that PRDX5 is effectively relocated to peroxisomes, lack of stronger signal in peroxisomes compared to cytoplasm may indicate that peroxisomal targeting of PRDX5 is insufficient to lead to accumulation of the enzyme in this organelle. Interestingly, study of the expression pattern of PRDX5 during mouse spinal cord development could not highlight colocalization of PRDX5 with catalase suggesting that peroxisomal targeting of PRDX5 might be weak at embryonic stages [25]. Though the slight overlap of signals associated to S-PRDX5 and ATPB might be an artefactual result, cytosolic S-PRDX5 signal being hard to distinguish from the complex three-dimensional structures formed by mitochondria, it might also be the result of leaking of the overexpressed S-PRDX5 into the mitochondria. Unexpected entry of cytosolic proteins into the mitochondria has previously been reported, notably for antioxidant enzyme SOD1 in transgenic mice overexpressing the protein [69]. Along these lines, mitochondria have been shown to act as guardians of cytosolic proteostasis (protein homeostasis) via the accumulation of overabundant proteins [70].

The model we propose here offers a unique and powerful tool to study function of PRDX5 in vivo and could yield valuable insight into the function of this enigmatic enzyme. For instance, this model could serve to evaluate the effect of L-PRDX5 and S-PRDX5 gain-offunction on biological processes taking place in the embryonic spinal cord, such as neurogenesis, differentiation, and migration of neuronal and glial cellular subtypes, or motor neuronal programmed cell death. In addition, targeting of specific cellular subtypes using gene promoters could be used to study the function of the enzyme in the context of a given cell type. For example, targeting of microglial cells would provide an opportunity to test hypotheses associated to the immune and inflammation-related functions of PRDX5. Furthermore, more general questions could also be approached, such as the degree of cytoprotection conveyed by this enzyme in live tissue subjected to oxidant or inflammatory stressors such as $\mathrm{H}_{2} \mathrm{O}_{2}$, organic peroxides, peroxynitrite, or LPS. Similarly, the impact of PRDX5 on redox-dependent signaling pathways would also be a question worthy of further investigation.

\section{Conclusion}

In this study, we report that though it is conserved throughout animal evolution, the PRDX5 gene appears to have been lost in birds. Identification of PRDX5 orthologs in non-avian amniotes shows disappearance of PRDX5 is specific to aves and enables us to date this genetic event to after the divergence of birds, 245 million years ago. Biological significance of loss of PRDX5 in birds remains elusive, but physiological and immunological differences in this class could be explanatory factors. Finally, using in ovo electroporation of long and short forms of human PRDX5, we report that subcellular localization mechanisms are conserved and highlight this technique as a valuable gain-of-function model for studying PRDX5 in vivo.

\section{Abbreviations}

ATPB: ATP synthase subunit beta; CAT: Catalase; E: Embryonic day; eGFP: Enhanced green fluorescent protein; HH: Hamilton-Hamburger stage; L-PRDX5: Long form of peroxiredoxin-5; MTS: Mitochondrial targeting 
sequence; PCD: Programmed cell death; PRDX: Peroxiredoxin; PTS: Peroxisomal targeting sequence; RNS: Reactive nitrogen species; ROS: Reactive oxygen species; S-PRDX5: Short form of peroxiredoxin-5

\section{Acknowledgements}

We acknowledge Agnès Roy and Frédéric Clotman for their help in learning the in ovo electroporation technique, and Stéphanie Debrulle for her help in optimizing the process. We also want to thank Valérie Van der Ecken for advice concerning sequence research, and Laure Bridoux for thorough reading and corrections to the manuscript, as well as her valuable insight and help throughout the study. Finally, thank you to Vicky Argyropoulou for archaeological excavation of frozen samples. Confocal microscopy was carried out in collaboration with the IMABIOL platform (ISV, Catholic University of Louvain)

\section{Funding}

This research was funded by the "Communauté française de Belgique Actions de Recherche Concertées (ARC)" \#10/15-026 of the "Direction générale de l'Enseignement non obligatoire et de la Recherche scientifique Direction de la Recherche scientifique - Communauté française de Belgique" and granted by the "Académie universitaire Louvain"; Grant sponsor: DIANE research program of the Walloon region; Grant number: 816844; Marc Pirson was holder of a FRIA fellowship (FRS-FNRS, Belgium). The aforementioned funding bodies only contributed financially to the study and did not take part in study, collection, analysis, or interpretation of data, or in the writing of the manuscript.

\section{Availability of data and materials}

Authors confirm that all data underlying the findings described in this manuscript are either fully available within the paper, or publicly available. Datasets and materials used during the current study are available from the corresponding author on reasonable request.

\section{Authors' contributions}

Conceived and designed the experiments: MP, BK Performed the experiments: MP, AC Analyzed the data: MP, AC, BK Wrote the manuscript: MP, BK Other: All authors read and approved the final manuscript.

\section{Ethics approval}

Experimental procedures on animals were approved by the animal ethics committee of the Université catholique de Louvain and are in agreement with the European directive 2010/63/UE.

\section{Consent for publication}

Not applicable.

\section{Competing interests}

The authors declare that they have no competing interests.

\section{Publisher's Note}

Springer Nature remains neutral with regard to jurisdictional claims in published maps and institutional affiliations.

\section{Received: 10 July 2017 Accepted: 31 January 2018}

\section{Published online: 08 February 2018}

\section{References}

1. Halliwell B, Gutteridge JMC. Free radicals in biology and medicine, 4th edn. Oxford. New York: Oxford University Press; 2007.

2. Hanschmann EM, Godoy JR, Berndt C, Hudemann C, Lillig CH. Thioredoxins, glutaredoxins, and peroxiredoxins-molecular mechanisms and health significance: from cofactors to antioxidants to redox signaling. Antioxid Redox Signal. 2013;19(13):1539-605.

3. Ray PD, Huang BW, Tsuji Y. Reactive oxygen species (ROS) homeostasis and redox regulation in cellular signaling. Cell Signal. 2012;24(5):981-90.

4. Cox AG, Winterbourn CC, Hampton MB. Measuring the redox state of cellular peroxiredoxins by immunoblotting. Methods Enzymol. 2010;474:51-66.

5. Hall A, Nelson K, Poole LB, Karplus PA. Structure-based insights into the catalytic power and conformational dexterity of peroxiredoxins. Antioxid Redox Signal. 2011;15(3):795-815.
6. Knoops B, Loumaye E, Van Der Eecken V. Evolution of the peroxiredoxins. Subcell Biochem. 2007:44:27-40.

7. Wood ZA, Schroder E, Robin Harris J, Poole LB. Structure, mechanism and regulation of peroxiredoxins. Trends Biochem Sci. 2003;28(1):32-40.

8. Manevich Y, Feinstein SI, Fisher AB. Activation of the antioxidant enzyme 1-CYS peroxiredoxin requires glutathionylation mediated by heterodimerization with pi GST. Proc Natl Acad Sci U S A. 2004;101(11):3780-5.

9. Ralat LA, Manevich $Y$, Fisher AB, Colman RF. Direct evidence for the formation of a complex between 1-cysteine peroxiredoxin and glutathione S-transferase pi with activity changes in both enzymes. Biochemistry. 2006; 45(2):360-72.

10. Ralat LA, Misquitta SA, Manevich Y, Fisher AB, Colman RF. Characterization of the complex of glutathione S-transferase pi and 1-cysteine peroxiredoxin. Arch Biochem Biophys. 2008;474(1):109-18.

11. Knoops B, Goemaere J, Van der Eecken V, Declercq JP. Peroxiredoxin 5: structure, mechanism, and function of the mammalian atypical 2-Cys peroxiredoxin. Antioxid Redox Signal. 2011:15(3):817-29.

12. Nguyen-Nhu NT, Berck J, Clippe A, Duconseille E, Cherif H, Boone C, Van de Eecken V, Bernard A, Banmeyer I, Knoops B. Human peroxiredoxin 5 gene organization, initial characterization of its promoter and identification of alternative forms of mRNA. Biochim Biophys Acta. 2007:1769(7-8):472-83.

13. Knoops B, Clippe A, Bogard C, Arsalane K, Wattiez R, Hermans C, Duconseille E, Falmagne $P$, Bernard A. Cloning and characterization of AOEB166, a novel mammalian antioxidant enzyme of the Peroxiredoxin family. J Biol Chem. 1999;274(43):30451-8.

14. Yamashita H, Avraham S, Jiang S, London R, Van Veldhoven PP, Subraman $\mathrm{S}$, Rogers RA, Avraham H. Characterization of human and murine PMP20 peroxisomal proteins that exhibit antioxidant activity in vitro. J Biol Chem. 1999;274(42):29897-904.

15. Van der Eecken V, Clippe A, Dekoninck S, Goemaere J, Walbreca G, Van Veldhoven PP, Knoops B. Abolition of peroxiredoxin-5 mitochondrial targeting during canid evolution. PLoS One. 2013;8(9):e72844.

16. Van der Eecken V, Clippe A, Van Veldhoven PP, Knoops B. Mitochondrial targeting of peroxiredoxin 5 is preserved from annelids to mammals but is absent in pig Sus Scrofa Domesticus. Mitochondrion. 2011;11(6):973-81.

17. Kent WJ, Sugnet CW, Furey TS, Roskin KM, Pringle TH, Zahler AM, Haussler D. The human genome browser at UCSC. Genome Res. 2002;12(6):9961006.

18. Gasteiger E, Gattiker A, Hoogland C, Ivanyi I, Appel RD, Bairoch A. ExPASy: the proteomics server for in-depth protein knowledge and analysis. Nucleic Acids Res. 2003:31(13):3784-8.

19. Wadley L, Sievers C, Bamford M, Goldberg P, Berna F, Miller C. Middle stone age bedding construction and settlement patterns at Sibudu, South Africa. Science. 2011;334(6061):1388-91.

20. Nicholas KB, Nicholas HB: GeneDoc: a tool for editing and annotating multiple sequence alignments. 1997.

21. Emanuelsson $\mathrm{O}$, Nielsen $\mathrm{H}$, Brunak S, von Heijne G. Predicting subcellular localization of proteins based on their $\mathrm{N}$-terminal amino acid sequence. J Mol Biol. 2000:300(4):1005-16.

22. Nielsen H, Engelbrecht J, Brunak S, von Heijne G. Identification of prokaryotic and eukaryotic signal peptides and prediction of their cleavage sites. Protein Eng. 1997;10(1):1-6.

23. Claros MG, Vincens P. Computational method to predict mitochondrially imported proteins and their targeting sequences. European journal of biochemistry / FEBS. 1996:241(3):779-86.

24. Goemaere J, Knoops B. Peroxiredoxin distribution in the mouse brain with emphasis on neuronal populations affected in neurodegenerative disorders. J Comp Neurol. 2012;520(2):258-80

25. Pirson M, Knoops B. Expression of peroxiredoxins and thioredoxins in the mouse spinal cord during embryonic development. J Comp Neurol. 2015 523(17):2599-617.

26. Pirson M, Debrulle S, Clippe A, Clotman F, Knoops B. Thioredoxin-2 modulates neuronal programmed cell death in the embryonic Chick spinal cord in basal and target-deprived conditions. PLoS One. 2015;10(11): e0142280

27. Wang MX, Wei A, Yuan J, Clippe A, Bernard A, Knoops B, Murrell GA. Antioxidant enzyme peroxiredoxin 5 is upregulated in degenerative human tendon. Biochem Biophys Res Commun. 2001;284(3):667-73.

28. Han JY, Song KD, Shin JH, Han BK, Park TS, Park HJ, Kim JK, Lillehoj HS, Lim $\mathrm{JM}$, Kim H. Identification and characterization of the peroxiredoxin gene family in chickens. Poult Sci. 2005;84(9):1432-8. 
29. Conrad M, Schick J, Angeli JP. Glutathione and thioredoxin dependent systems in neurodegenerative disease: what can be learned from reverse genetics in mice. Neurochem Int. 2013;62(5):738-49.

30. Fourquet $\mathrm{S}$, Huang ME, D'Autreaux B, Toledano MB. The dual functions of thiol-based peroxidases in $\mathrm{H}_{2} \mathrm{O} 2$ scavenging and signaling. Antioxid Redox Signal. 2008;10(9):1565-76.

31. Hamilton RT, Walsh ME, Van Remmen H. Mouse Models of Oxidative Stress Indicate a Role for Modulating Healthy Aging. Journal of clinical \& experimental pathology, Suppl. 2012:4.

32. Peng Y, Yang PH, Ng SS, Lum CT, Kung HF, Lin MC. Protection of Xenopus Laevis embryos against alcohol-induced delayed gut maturation and growth retardation by peroxiredoxin 5 and catalase. J Mol Biol. 2004;340(4):819-27.

33. Ezcurra MD, Scheyer TM, Butler RJ. The origin and early evolution of Sauria: reassessing the permian saurian fossil record and the timing of the crocodile-lizard divergence. PLoS One. 2014;9(2):e89165.

34. Zhang G, Li C, Li Q, Li B, Larkin DM, Lee C, Storz JF, Antunes A, Greenwold MJ, Meredith RW, et al. Comparative genomics reveals insights into avian genome evolution and adaptation. Science. 2014;346(6215):1311-20.

35. Lovell PV, Wirthlin M, Wilhelm L, Minx P, Lazar NH, Carbone L, Warren WC, Mello CV. Conserved syntenic clusters of protein coding genes are missing in birds. Genome Biol. 2014:15(12):565.

36. Backstrom N, Forstmeier W, Schielzeth H, Mellenius H, Nam K, Bolund E, Webster MT, Ost T, Schneider M, Kempenaers B, et al. The recombination landscape of the zebra finch Taeniopygia Guttata genome. Genome Res. 2010;20(4):485-95.

37. Hickey AJ, Jullig M, Aitken J, Loomes K, Hauber ME, Phillips AR. Birds and longevity: does flight driven aerobicity provide an oxidative sink? Ageing Res Rev. 2012;11(2):242-53.

38. Munshi-South J, Wilkinson GS. Bats and birds: exceptional longevity despite high metabolic rates. Ageing Res Rev. 2010;9(1):12-9.

39. Ogburn CE, Austad SN, Holmes DJ, Kiklevich JV, Gollahon K, Rabinovitch PS, Martin GM. Cultured renal epithelial cells from birds and mice: enhanced resistance of avian cells to oxidative stress and DNA damage. J Gerontol A Biol Sci Med Sci. 1998;53((4):B287-92.

40. Pamplona R, Portero-Otin M, Requena JR, Thorpe SR, Herrero A, Barja G. A low degree of fatty acid unsaturation leads to lower lipid peroxidation and lipoxidation-derived protein modification in heart mitochondria of the longevous pigeon than in the short-lived rat. Mech Ageing Dev. 1999;106(3):283-96.

41. Pamplona R, Portero-Otin M, Riba D, Ledo F, Gredilla R, Herrero A, Barja G. Heart fatty acid unsaturation and lipid peroxidation, and aging rate, are lower in the canary and the parakeet than in the mouse. Aging. 1999;11(1):44-9.

42. Barja G, Herrero A. Localization at complex I and mechanism of the higher free radical production of brain nonsynaptic mitochondria in the short-lived rat than in the longevous pigeon. J Bioenerg Biomembr. 1998;30(3):235-43.

43. Herrero A, Barja G. Sites and mechanisms responsible for the low rate of free radical production of heart mitochondria in the long-lived pigeon. Mech Ageing Dev. 1997;98(2):95-111.

44. Herrero A, Barja G. H2O2 production of heart mitochondria and aging rate are slower in canaries and parakeets than in mice: sites of free radical generation and mechanisms involved. Mech Ageing Dev. 1998;103(2):133-46.

45. Brunet-Rossinni AK. Reduced free-radical production and extreme longevity in the little brown bat (Myotis Lucifugus) versus two non-flying mammals. Mech Ageing Dev. 2004;125(1):11-20.

46. Wilhelm Filho D, Althoff SL, Dafre AL, Boveris A. Antioxidant defenses, longevity and ecophysiology of south American bats. Comparative biochemistry and physiology Toxicology \& pharmacology : CBP. 2007;146(1-2):214-20.

47. Simmons NB, Seymour KL, Habersetzer J, Gunnell GF. Primitive early Eocene bat from Wyoming and the evolution of flight and echolocation. Nature. 2008;451(7180):818-21.

48. Boardman PE, Sanz-Ezquerro J, Overton IM, Burt DW, Bosch E, Fong WT, Tickle C, Brown WR, Wilson SA, Hubbard SJ. A comprehensive collection of chicken cDNAs. Current biology : CB. 2002;12(22):1965-9.

49. Caldwell RB, Kierzek AM, Arakawa H, Bezzubov Y, Zaim J, Fiedler P, Kutter S, Blagodatski A, Kostovska D, Koter M, et al. Full-length cDNAs from chicken bursal lymphocytes to facilitate gene function analysis. Genome Biol. 2005;6(1):R6

50. Kong BW, Kim H, Foster DN. Cloning and expression analysis of chicken phospholipid-hydroperoxide glutathione peroxidase. Anim Biotechnol. 2003; 14(1):19-29

51. Savolainen P, Fitzsimmons C, Arvestad L, Andersson L, Lundeberg J. ESTs from brain and testis of white leghorn and red junglefowl: annotation, bioinformatic classification of unknown transcripts and analysis of expression levels. Cytogenetic and genome research. 2005;111(1):79-87.
52. Barja G, Cadenas S, Rojas C, Lopez-Torres M, Perez-Campo R. A decrease of free radical production near critical targets as a cause of maximum longevity in animals. Comparative biochemistry and physiology Biochemistry and molecular biology. 1994;108(4):501-12.

53. Lopez-Torres M, Perez-Campo R, Rojas C, Cadenas S, Barja G. Maximum life span in vertebrates: relationship with liver antioxidant enzymes, glutathione system, ascorbate, urate, sensitivity to peroxidation, true malondialdehyde, in vivo $\mathrm{H}_{2} \mathrm{O} 2$, and basal and maximum aerobic capacity. Mech Ageing Dev. 1993;70(3):177-99.

54. Perez-Campo R, Lopez-Torres M, Rojas C, Cadenas S, Barja G. Longevity and antioxidant enzymes, non-enzymatic antioxidants and oxidative stress in the vertebrate lung: a comparative study. Journal of comparative physiology $B$, Biochemical, systemic, and environmental Physiology. 1994;163(8):682-9.

55. Abbas K, Breton J, Picot CR, Quesniaux V, Bouton C, Drapier JC. Signaling events leading to peroxiredoxin 5 up-regulation in immunostimulated macrophages. Free Radic Biol Med. 2009;47(6):794-802.

56. Sun HN, Kim SU, Huang SM, Kim JM, Park YH, Kim SH, Yang HY, Chung KJ, Lee $\mathrm{TH}$, Choi HS, et al. Microglial peroxiredoxin $\mathrm{V}$ acts as an inducible antiinflammatory antioxidant through cooperation with redox signaling cascades. J Neurochem. 2010;114(1):39-50.

57. Shichita T, Hasegawa E, Kimura A, Morita R, Sakaguchi R, Takada I, Sekiya T, Ooboshi $\mathrm{H}$, Kitazono T, Yanagawa $\mathrm{T}$, et al. Peroxiredoxin family proteins are key initiators of post-ischemic inflammation in the brain. Nat Med. 2012; 18(6):911-7.

58. Radyuk SN, Michalak K, Klichko VI, Benes J, Orr WC. Peroxiredoxin 5 modulates immune response in drosophila. Biochim Biophys Acta. 2010; 1800(11):1153-63.

59. Kaiser P, Poh TY, Rothwell L, Avery S, Balu S, Pathania US, Hughes S, Goodchild M, Morrell S, Watson M, Bumstead N, Kaufman J, Young JR. A Genomic Analysis of Chicken Cytokines and Chemokines. Journal of Interferon \& Cytokine Research. 2005;25(8):467-484.

60. Magor KE, Miranzo Navarro D, Barber MR, Petkau K, Fleming-Canepa X, Blyth $\mathrm{GA}$, Blaine $\mathrm{AH}$. Defense genes missing from the flight division. Dev Comp Immunol. 2013;41(3):377-88.

61. Banmeyer I, Marchand C, Verhaeghe C, Vucic B, Rees JF, Knoops B. Overexpression of human peroxiredoxin 5 in subcellular compartments of Chinese hamster ovary cells: effects on cytotoxicity and DNA damage caused by peroxides. Free Radic Biol Med. 2004;36(1):65-77.

62. Leyens G, Donnay I, Knoops B. Cloning of bovine peroxiredoxins-gene expression in bovine tissues and amino acid sequence comparison with rat, mouse and primate peroxiredoxins. Comparative biochemistry and physiology Part B, Biochemistry \& molecular biology. 2003;136(4):943-55

63. Li J, Li L, Zhang S, Zhang G. Cloning, genomic structure, and expression analysis of peroxiredoxin $V$ from bay scallop Argopecten Irradians. Fish \& shellfish immunology. 2011;30(1):309-16.

64. Oberley TD, Verwiebe E, Zhong W, Kang SW, Rhee SG. Localization of the thioredoxin system in normal rat kidney. Free Radic Biol Med. 2001;30(4):412-24

65. Pagliarini DJ, Calvo SE, Chang B, Sheth SA, Vafai SB, Ong SE, Walford GA Sugiana C, Boneh A, Chen WK, et al. A mitochondrial protein compendium elucidates complex I disease biology. Cell. 2008;134(1):112-23.

66. Radyuk SN, Michalak K, Klichko VI, Benes J, Rebrin I, Sohal RS, Orr WC. Peroxiredoxin 5 confers protection against oxidative stress and apoptosis and also promotes longevity in drosophila. The Biochemical journal. 2009; 419(2):437-45.

67. Zhang S, Wang $C$, Wang $Y$, Wei R, Jiang G, Ju H. Presence and characterization of complement-like activity in the amphioxus Branchiostoma Belcheri Tsingtauense. Zool Sci. 2003;20(10):1207-14.

68. Nakamura H, Katahira T, Sato T, Watanabe Y, Funahashi J. Gain- and loss-offunction in chick embryos by electroporation. Mech Dev. 2004;121(9):1137-43.

69. Tafuri F, Ronchi D, Magri F, Comi GP, Corti S. SOD1 misplacing and mitochondrial dysfunction in amyotrophic lateral sclerosis pathogenesis. Front Cell Neurosci. 2015;9:336

70. Ruan L, Zhou C, Jin E, Kucharavy A, Zhang Y, Wen Z, Florens L, Li R. Cytosolic proteostasis through importing of misfolded proteins into mitochondria. Nature. 2017;543(7645):443-6.

71. Declercq JP, Evrard C, Clippe A, Stricht DV, Bernard A, Knoops B. Crystal structure of human peroxiredoxin 5, a novel type of mammalian peroxiredoxin at 1.5 a resolution. J Mol Biol. 2001:311(4):751-9. 